\title{
Functional significance of nuclear export and mRNA binding of meiotic regulator Spo5 in fission yeast
}

Naoyuki Togashi ${ }^{1,2}$, Akira Yamashita ${ }^{1}$, Masamitsu Sato ${ }^{3}$ and Masayuki Yamamoto ${ }^{1,2,4^{*}}$

\begin{abstract}
Background: Meiotic cells undergo two rounds of nuclear division and generate gametes. Previous studies have indicated that a number of transcription factors modulate the transcriptome in successive waves during meiosis and spore formation in fission yeast. However, the mechanisms underlying the post-transcriptional regulation in meiosis are not fully understood. The fission yeast $\mathrm{spo5}^{+}$gene encodes a meiosis-specific RNA-binding protein, which is required for the progression of meiosis II and spore formation. However, the target RNA molecules of Spo5 are yet to be identified. Characterization of meiosis-specific RNA-binding proteins will provide insight into how post-transcriptional regulation influence gene expression during sexual differentiation.

Results: To assess the functional significance of RNA-recognition motifs (RRMs) of Spo5, we constructed a series of new spo5 truncated mutants and previously reported spo5 missense mutants. In addition, we isolated novel spo5 missense mutants. The phenotypic characteristics of these mutants indicated that the RRMs are essential for both the localization and function of the protein. Interestingly, Spo5 is exported from the nucleus to the cytoplasm via the Rae1-dependent mRNA export pathway, but is unlikely to be involved in global mRNA export. Furthermore, cytoplasmic localization of Spo5 is important for its function, which suggests the involvement of Spo5 in post-transcriptional regulation. We identified $p \mathrm{Cr}^{+}{ }^{+} \mathrm{mRNA}$ as one of the critical targets of Spo5. The $p c r 1^{+}$gene encodes an activating transcription factor/CAMP response element binding (ATF/CREB) transcription factor family. Among the four family members, namely Pcr1, Atf1, Atf21, and Atf31, only the mRNA encoding Pcr1 binds to Spo5.

Conclusions: Spo5 is exported from the nucleus with mRNAs via the Rae1-dependent pathway. RRMs are necessary for this process and also for the function of Spo5 after the nuclear export. Spo5 appears to influence the activity of $p c r 1^{+}$ mRNA, and the mechanism of how Spo5 stimulates the mRNA to promote the progression of meiosis II and spore formation remains an intriguing question for future research.
\end{abstract}

Keywords: Fission yeast, Meiosis, RNA export, RNA-binding protein, ATF/CREB family

\section{Background}

Meiosis is a specialized cell division process, which includes premeiotic DNA synthesis, DNA recombination followed by two rounds of cell division, and gametogenesis [1-4]. It has been shown in the fission yeast Schizosaccharomyces pombe [5-8] and the budding yeast Saccharomyces cerevisiae [1,9-12] that a number of transcription factors

\footnotetext{
* Correspondence: yamamoto@nibb.ac.jp

${ }^{1}$ Kazusa DNA Research Institute, 2-6-7 Kazusa-kamatari, Kisarazu, Chiba 292-0818, Japan

${ }^{2}$ Department of Biophysics and Biochemistry, Graduate School of Science, University of Tokyo, 7-3-1 Hongo, Tokyo 113-0033, Japan

Full list of author information is available at the end of the article
}

dramatically modulate the transcriptome to facilitate meiosis, thereby playing critical roles in meiotic progression and sporulation (gametogenesis). In addition to transcriptional regulation, post-transcriptional regulation plays a fundamental role in the progression of meiosis and gametogenesis in higher eukaryotes. For example, cessation of transcription followed by complex translational activation and repression of stored maternal mRNAs occurs in Xenopus oocytes during meiotic progression (oocyte maturation) [13]. In fission yeast, a specialized regulation of meiosis, called selective elimination of meiosis-specific mRNAs, facilitates the post-transcriptional degradation of meiotic mRNAs during the mitotic cell cycle [14]. We 
were interested in elucidating any additional posttranscriptional regulation that might contribute to the dynamic changes observed in gene expression during meiosis in the fission yeast.

The spo5 mutant was isolated as a sporulation-deficient mutant in the original genetic screen of defective mutants in meiotic progression and/or sporulation, performed nearly half a century ago [15]. Previous studies have indicated that the $s p o 5^{+}$gene encodes a meiosis-specific RNAbinding protein, carrying two RNA-recognition motifs (RRMs) in the C-terminal part (aas 192-567), and regulates the progression of meiosis II and spore formation [16-20]. Although it seems apparent that Spo5 plays an essential role to coordinate meiosis and sporulation, controlling a number of targets, the RNA molecules that bind to Spo5 have not yet been identified, except for our recent finding that $c d c 13^{+}$mRNA, encoding cyclin $\mathrm{B}$, can do so [21]. Because Spo5 is likely to be involved in posttranscriptional events during meiosis, such as pre-mRNA processing, mRNA export, translation, and mRNA degradation, we analyzed this RNA-binding protein to evaluate its functions.

In this report, we demonstrate that the RRMs on Spo5 are essential for its cytoplasmic localization, where it exerts its function. Spo5 appears to be exported from the nucleus to the cytoplasm through the Rae1-dependent mRNA export pathway, but is unlikely to be involved in general mRNA export. We also show that one of the critical target RNA molecules for Spo5 is $p c r 1^{+}$mRNA. Pcr1 belongs to the ATF/CREB family of transcription factors, which consists of four members in fission yeast: Pcr1, Atf1, Atf21, and Atf31. The functional relationship between Spo5 and Pcr1 is also analyzed.

\section{Results and discussion}

\section{RNA recognition motifs are essential for the localization} and function of Spo5

First, we evaluated the mechanism underlying the subcellular localization of Spo5. A previous study suggested that the C-terminal half of Spo5 is required for its cytoplasmic localization [19]. As this region contains the RRMs, we examined whether these might be responsible for the localization of Spo5. We constructed a series of truncation mutants that lacked the RRM region (Figure 1A). Each mutant carried the mutated spo5 gene in place of the wild-type gene on the chromosome, and expressed the mutant protein from the authentic spo5 promoter. All truncated mutant proteins that lacked at least one RRM showed abnormal nuclear accumulation, as indicated by the fluorescent signals of fused green fluorescent protein (GFP) (Figure 1A). We confirmed that mutant proteins were produced in a comparable amount to wild-type Spo5, by examining the shortest mutant Spo5(1-296) and the RRM2-deletion mutant, although the protein level might be slightly lower in the latter case (Additional 1: Figure S1). The RRM truncation mutants were defective in sporulation (Figure 1B), suggesting that the existence of intact RRMs is correlated with the cytoplasmic localization and proper function of Spo5. While we could not exclude the possibility that some of the effect of the mutations on sporulation might be due to lower levels of the mutant proteins, the effect on sporulation was apparently more dramatic than the effect on protein levels, supporting the importance of the RRMs/cytoplasmic localization in Spo5 function.

There was one exception, namely Spo5(1-456)-GFP, which has two RRMs but lacks the C-terminal region. Although it localized mostly to the cytoplasm, it was not functional, whereas Spo5(1-525)-GFP, which has two RRMs and an additional C-terminal portion, was functional (Figure 1A,B). This indicates that, in addition to intact RRMs, the C-terminal region adjacent to the second RRM is essential for the Spo5 function.

To clarify whether RNA-binding is required for Spo5 localization and function, we introduced missense mutations to the conserved motifs in RRMs. We constructed mutant strains that were similar to the F341A and F427A mutations previously analyzed by Kasama and colleagues [19]. All of the mutant proteins, namely Spo5(F341A)GFP, Spo5(F427A)-GFP, and Spo5(F341A, F427A)-GFP, accumulated in the nucleus, as did the RRM-truncated proteins (Figure 1C). These mutants were also deficient in sporulation (Figure 1D). Similar, or slightly lower production of Spo5(F341A, F427A) protein compared to the wild-type was confirmed (Additional file 1: Figure S1). The nuclear localization of these mutated Spo5-GFP proteins in our study was somewhat different from that observed in the previous study, which reported that Spo5(F341A, F427A)-GFP shows a punctate distribution in both the cytoplasm and nucleus [19]. Our precise analysis suggested that Spo5(F341A, F427A) protein might exhibit punctate cytoplasmic distribution during early meiotic stages such as the horsetail-movement and one-nucleus stages (Additional file 2: Figure S2). The apparent difference in localization may also reflect changes in the strain construction: In the previous study, the spo5 mutant allele was integrated into the chromosomal leu1 locus, whereas the mutant allele was integrated in the authentic spo5 locus in our case. However, the actual reasons remain unclear.

We also performed a genetic screen for novel missense mutants of spo5 that were defective in meiosis and sporulation, and isolated more than ten different mutants including spo5(F341L) and spo5(S365P), both of which contained a mutation in the RRM domain. When assayed at $30^{\circ} \mathrm{C}$, Spo5(F341L)-GFP accumulated in the nucleus as did Spo5(F341A)-GFP, but Spo5(S365P)-GFP did not, showing a unique phenotype (Figure 1C). Because 

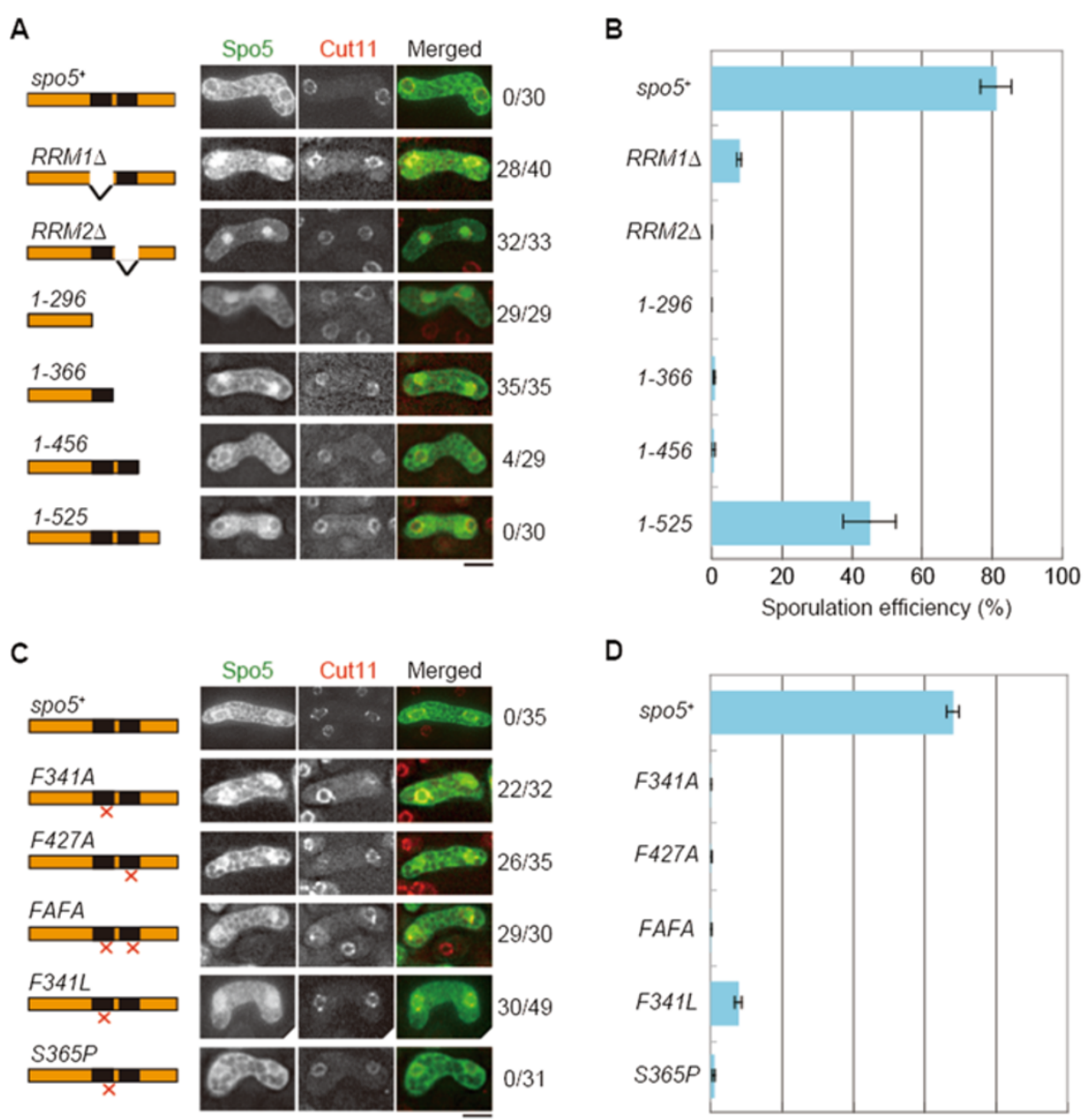

D

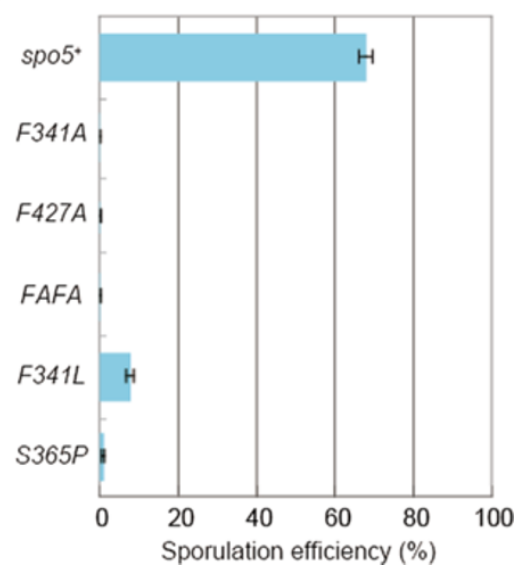

Figure 1 RNA recognition motifs are essential for the localization and function of Spo5. (A) Localization analyses using truncated mutants of Spo5. Localization of wild-type and mutant proteins of Spo5-GFP (green) during the period between meiosis I and meiosis II was detected using the nuclear envelope marker Cut11-4mRFP (red). Numbers on the right indicate the frequency of cells displaying nuclear GFP signals. Schematic images depict the domains of the mutant proteins. Black boxes depict two RNA-recognition motifs, RRM1 and RRM2, respectively. Scale bar, $5 \mu \mathrm{m}$. (B) Sporulation efficiency of specific mutants used in (A), measured at $30^{\circ} \mathrm{C}(\mathrm{n}>500)$. Error bars indicate standard deviation. (C) Localization analyses using point mutants of Spo5. Localization of Spo5-GFP harboring specific point mutations was observed as in (A). Positions of mutation sites $(X)$ are shown in schematic images. Scale bar, $5 \mu \mathrm{m}$. (D) Sporulation efficiency of the strains used in (C), measured at $30^{\circ} \mathrm{C}(n>500)$. Error bars indicate standard deviation.

aromatic residues in the RRM, such as F341, have been shown to interact directly with RNA [22], we speculated that the RNA-binding of Spo5 might be important for its cytoplasmic localization.

\section{Spo5 localizes to the cytoplasm via the mRNA export pathway}

RRMs appear to be essential for the translocation of Spo5 from the nucleus to the cytoplasm. Therefore, we investigated whether Spo5 might be exported to the cytoplasm via the mRNA export machinery using a mutant of the mRNA export factor, Rae1. The Rae1dependent pathway is conserved from the budding yeast [23] to humans [24]. The fission yeast rae1-167 mutant exhibited defective mRNA export at restrictive temperatures [25]. We induced meiosis in wild-type (WT) and rae1-167 cells at $25^{\circ} \mathrm{C}$, the latter of which produced Spo5 protein in a comparable or slightly lower amount (Additional file 1: Figure S1), and transferred the cells to $36^{\circ} \mathrm{C}$ to inactivate Rae1-167. In WT cells, Spo5-GFP was mainly localized to the cytoplasm both at $25^{\circ} \mathrm{C}$ and $36^{\circ} \mathrm{C}$ (Figure 2A). By contrast, Spo5-GFP accumulated in the nucleus in rae1-167 cells at the restrictive temperature (Figure 2A). This suggests that Spo5 is likely to be exported to the cytoplasm via the mRNA export machinery. Consistent with this idea, Spo5 accumulated in the nucleus when mRNA synthesis was inhibited by the addition of 1,10-phenanthroline (Figure 2B,C). We also 


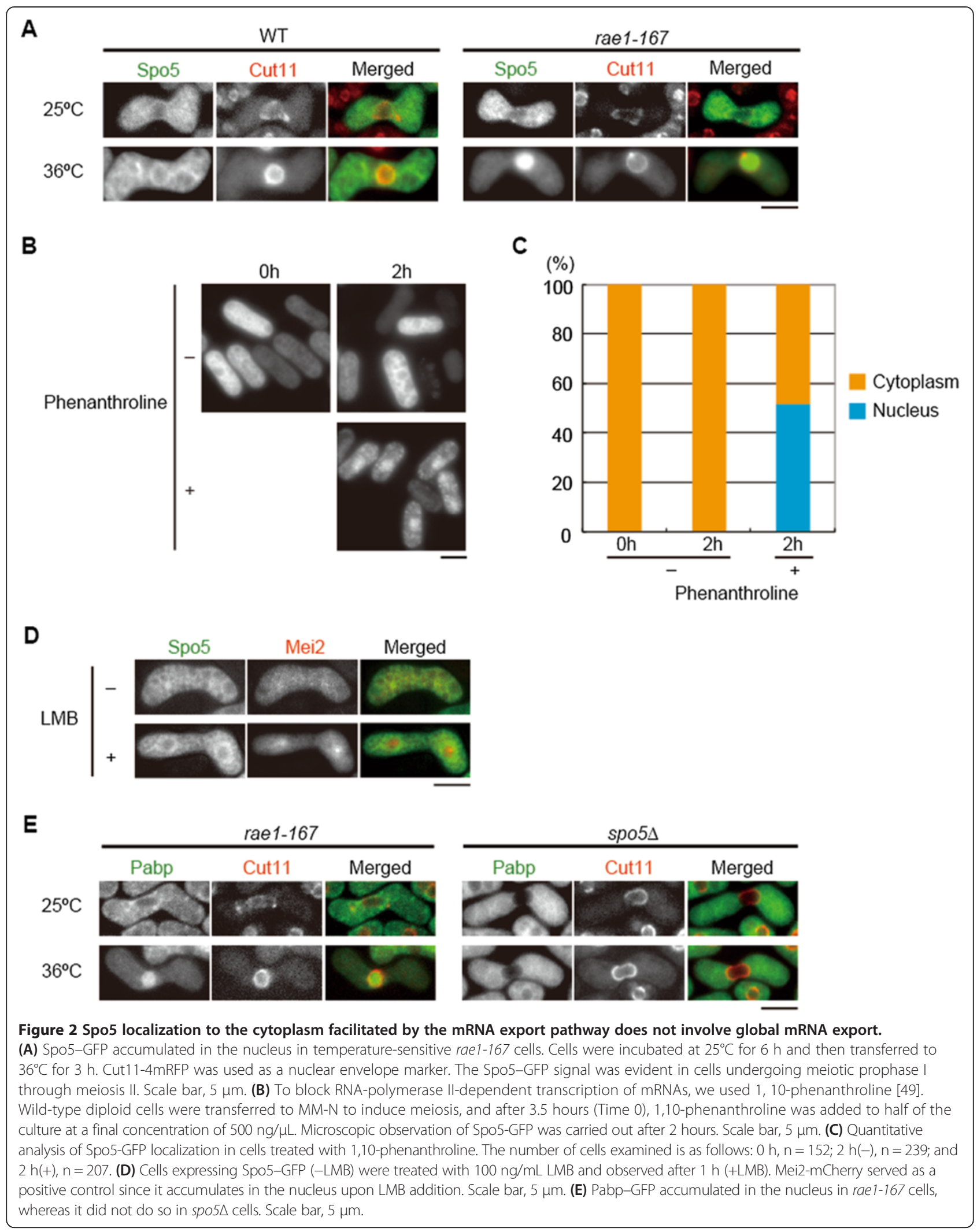


noticed that sporulation was markedly inefficient in rae1-167 cells (spo5-GFP rae $1^{+}$, $83 \%$ vs. spo5-GFP rae1$167,19 \%)$, indicating that Rae1 plays an important role in meiotic progression and sporulation.

Next, we investigated the possible involvement of the exportin-mediated mRNA export pathway [26] in the localization of Spo5. Ran GTPase is known as the major organizer of importin/exportin-mediated nucleocytoplasmic transport [27]. When the Ran-exportin nuclear export pathway was blocked with leptomycin B (LMB), a potent inhibitor of exportin/Crm1 [28], Spo5-GFP remained in the cytoplasm (Figure 2D), demonstrating that exportin/Crm1 is dispensable for the export of Spo5.

To examine whether Spo5 itself is a component of the mRNA transport machinery, we monitored the localization of the poly (A)-binding protein (Pabp), which has been shown to accumulate in the nucleus in the rae1-167 mutant [29]. Pabp-GFP was found to localize in the cytoplasm in spo5 $\Delta$ cells (Figure 2E), suggesting that Spo5 is not an mRNA export factor.

Taken together, we conclude that Spo5 is exported to the cytoplasm via the mRNA export machinery, but is unlikely to be involved in global mRNA export. It was recently shown that Drosophila RAE1 plays an essential role in male meiosis and spermatogenesis [30]. Fission yeast Rae1 may also promote meiosis by transporting mRNPs from the nucleus to the cytoplasm. Some RNAbinding proteins are also known to be exported to the cytoplasm through binding to mRNAs [31-33]. The mRNA-dependent nuclear export of RNA-binding proteins may emerge as a more universal phenomenon among eukaryotes than previously anticipated.

\section{Nuclear export is important for the function of Spo5}

The findings of this study indicated that mutations in RRMs caused abnormal nuclear accumulation and loss of function of Spo5. This suggests that the mRNAdependent nuclear export of Spo5 is required for its function, although it is also possible that the loss of RNA-binding in Spo5 resulted in its abnormal localization as a secondary effect. Hence, we employed two approaches to investigate whether the nuclear export of Spo5 is a fundamental requirement for its function.

First, we used an external nuclear localization signal (NLS) to alter the localization of Spo5 artificially from the cytoplasm to the nucleus. Although the addition of NLS appeared to make the protein less stable (Additional file 1: Figure S1), cells expressing Spo5-NLS-GFP accumulated the fusion proteins detectably in the nucleus, indicating that the NLS sequence was functional (Figure 3A). The sporulation efficiency of these cells was significantly reduced, and they produced abnormally shaped spores or asci with less than four spores (Figure 3B,C). While the destabilization of Spo5-NLS was likely to contribute largely to the sporulation defect, enforced nuclear migration of Spo5 also appeared to abolish its function to promote meiosis and sporulation, suggesting that the nuclear export of Spo5 is important for its optimal function. The viability of the spores generated in spo5-NLS-GFP cells was comparable to that of the control (spo5-GFP, 50\% vs. spo5-NLS-GFP 40\%), implying that the loss of Spo5 function did not affect the germination potential.

Second, we investigated whether artificial nuclear export of Spo5 could suppress sporulation defects in some spo5 mutants. Removal of RRM1 caused nuclear accumulation of Spo5 and sporulation defects, although a small portion of spo5 RRM1 $\Delta$ cells could sporulate (Figure 1A,B). The fusion of a nuclear export signal (NES) to Spo5RRM1 1 (Spo5RRM1 $\Delta$-NES-GFP) enhanced its localization to the cytoplasm (Figure 3D). However, the sporulation efficiency was much lower than that observed for Spo5-GFP carrying intact RRM1, and was only comparable to that observed for Spo5RRM1 $\Delta$-GFP (Figure 3E). Hence, we conclude that the RNA-binding activity of Spo5 is necessary not only for nuclear export of the Spo5mRNA complex via the mRNA export machinery, but also for the function of Spo5 in the cytoplasm.

\section{$p c r 1^{+}$mRNA is one of the critical targets of Spo5}

The spo5(S365P) mutant isolated in this study was unique in that it did not accumulate Spo5(S365P) protein efficiently in the nucleus in rae1-167 mutant cells at the restrictive temperature (Additional file 3: Figure S3A), although it was produced in a comparable or slightly lower amount to the wild-type (Additional file 1: Figure S1), implying that this mutant protein might be defective in nuclear import. In addition, the spo5(S365P) mutant was less leaky compared to the spo5(F341L) mutant, which sporulated almost normally at $25^{\circ} \mathrm{C}$, as indicated by iodine staining [15] and microscopic measurement (Additional file 3: Figure S3B, C). Therefore, we screened for multi-copy suppressors of spo5 $(S 365 P)$, expecting to identify novel candidates. We introduced the cDNA library to the spo5(S365P) mutant and picked up iodine-positive colonies. In this screening we obtained clones encoding transcription factors. Among them was the $p c r 1^{+}$gene, which encodes an ATF/CREB family transcription factor. The $p c r 1^{+}$gene was not a novel candidate, because we have previously reported the isolation of this gene as a multicopy suppressor of the spo5 mutant [34]. We and others have characterized Pcr1, which is now known to form a heterodimer with Atf1 and binds to the ade6-M26 meiotic recombination hotspot $[35,36]$.

The relationship between Pcr1 and Spo5, however, has not been delineated. Transcription of $\mathrm{pcr}^{+}$was not lowered in the spo5 null mutant, spo5 $\Delta$ (Additional file 4: Figure S4A). The levels of $p c r 1^{+}$transcripts gradually 

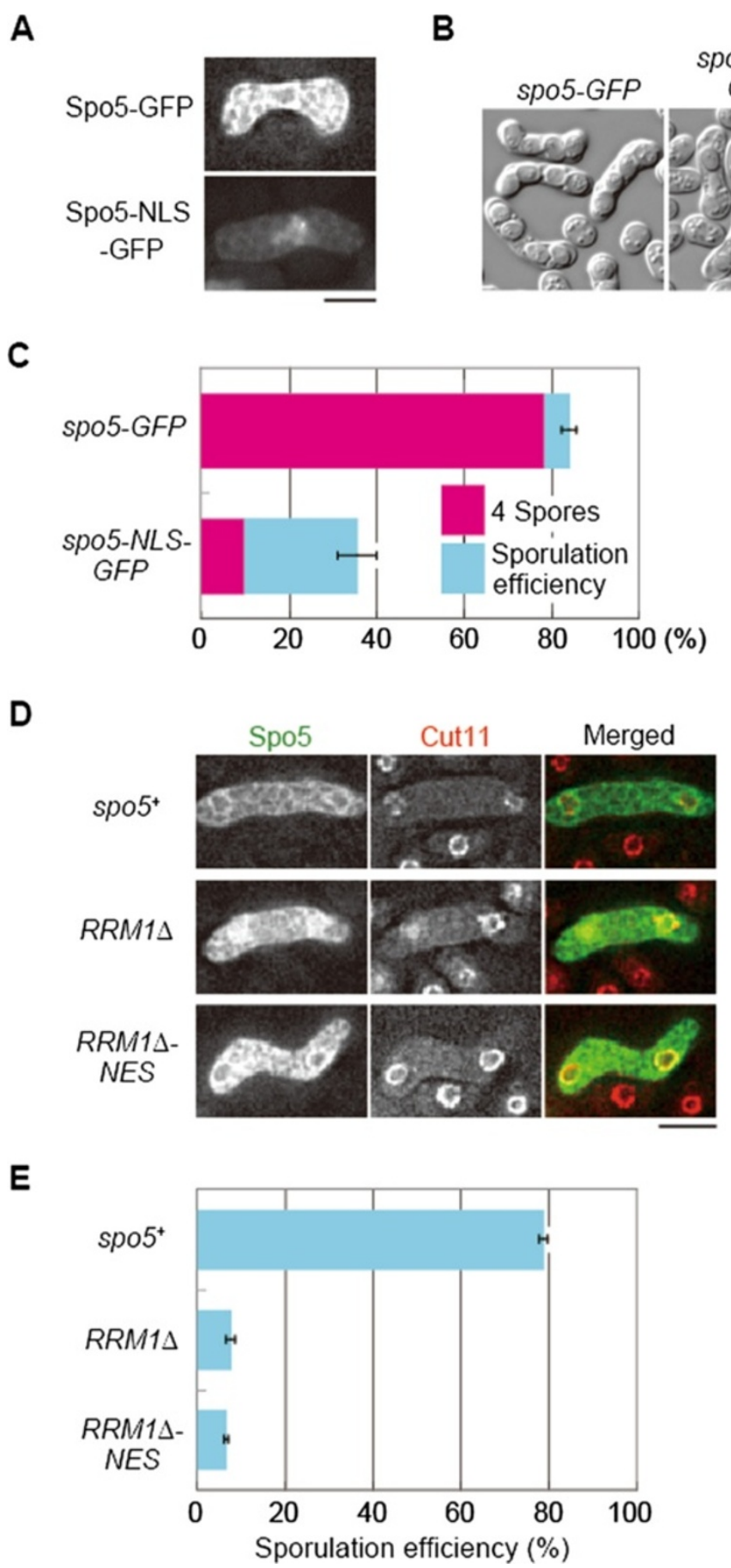

Figure 3 Nuclear export is important for the function of Spo5. (A) Spo5 fused with an NLS sequence derived from SV40 large T antigen and GFP (Spo5-NLS-GFP) accumulated in the nucleus during meiosis. Scale bar, 5 mm. (B) Addition of the NLS caused deficient sporulation. Differential interference contrast (DIC) images are shown. Scale bar, 5 mm. (C) Quantitative representation of the reduction in sporulation efficiency (light blue) and the frequency of four-spore asci (magenta) $(n>500)$. Error bars indicate standard deviation. (D) Nuclear accumulation of Spo5 (RRM1 $\triangle$ )-GFP was suppressed by the fusion of the NES sequence. Scale bar, $5 \mu \mathrm{m}$. (E) Addition of NES did not suppress the sporulation defects of $\operatorname{Spo5}(\operatorname{RRM} 1 \Delta)(n>500)$. Error bars indicate standard deviation.

increase toward late meiosis [5] (after $4 \mathrm{~h}$ in Additional file 4: Figure S4A), suggesting that Pcr1 may also function in a meiotic process other than meiotic recombination.
Overexpression of $\mathrm{pcr}^{+}$could suppress the sporulation deficiency of spo5(S365P) and other spo5 mutants including spo5 $\Delta$ (Figure 4A,B). These data suggest that Pcr1 may 
A

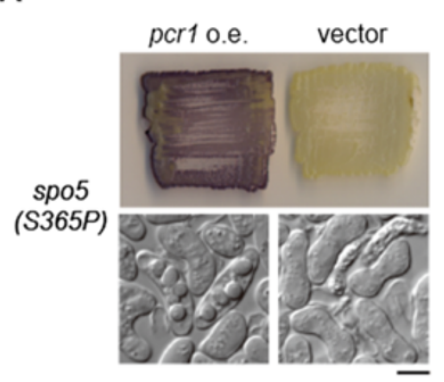

C

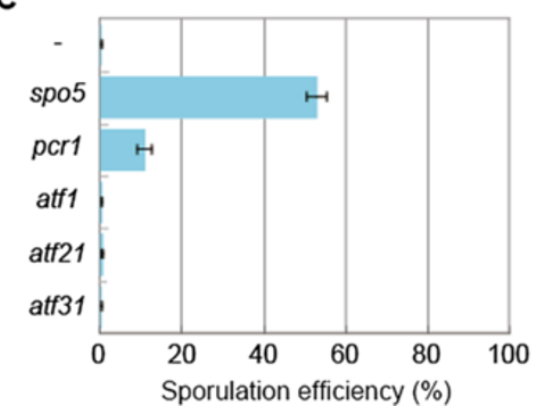

B

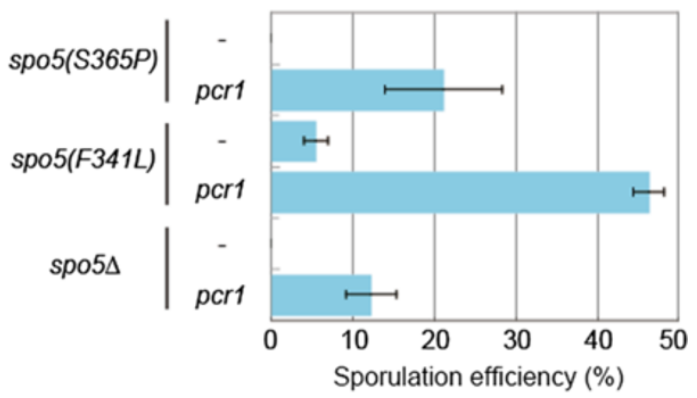

Figure $4 \mathrm{pcr}^{+}$mRNA is one of the critical targets of Spo5. (A) The sporulation of the spo5(S365P) strain was detected by dark brown staining with iodine vapor when $\mathrm{C} \mathrm{Cr}^{+}$was overexpressed. Stained patches and DIC images of the cells are shown. Scale bar, 5 Hm. (B) Sporulation defects of

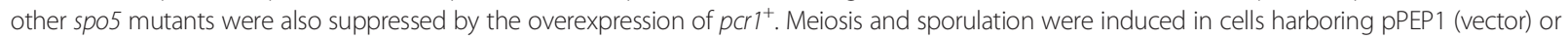
pPEP3-pcr $7^{+}$, and the sporulation efficiency was calculated $(n>500)$. Error bars indicate standard deviation. (C) Meiosis was induced in $s p 05 \Delta$ cells harboring plasmids containing $s p o 5^{+}, p c r 1^{+}, a t f 1^{+}, a t f 21^{+}$, and $a t f 31^{+}$, or the empty vector on $\mathrm{SSA}$ at $30^{\circ} \mathrm{C}$ for 3 days, and sporulation efficiency was calculated $(n>500)$. Error bars indicate standard deviation.

act downstream of Spo5, and that meiotic progression can be promoted substantially without Spo5 if Pcr1 is expressed at sufficiently high levels.

S. pombe has four ATF/CREB family proteins, namely Pcr1, Atf1, Atf21, and Atf31 [37]. Although we examined all of them, only Pcr1 could suppress sporulation defects of spo5 $\Delta$ cells when overexpressed (Figure 4C). Curiously, overexpression of Atf1, a functional partner that forms a heterodimer with Pcr1 [35], failed to suppress spo5 mutants. This suggested that Spo5 might be specifically related to $\mathrm{crr}^{+}$gene expression, and furthermore, that Pcr1 might promote transcription of some meiotic genes that are regulated by Spo5. It was also evident that Spo5 could regulate factors other than Pcr1 that are important for meiotic progression and sporulation, because Pcr1 could not suppress sporulation defects of spo5 $\Delta$ as efficiently as Spo5 itself (Additional file 4: Figure S4B), although $\mathrm{pcrl}^{+}$overexpression increased the amount of $p c r 1^{+}$mRNA in spo5 $\Delta$ cells to a higher level than $s p o 5^{+}$ overexpression (Additional file 4: Figure S4C). Indeed, we have recently demonstrated that Spo5 is necessary to maintain proper expression of cyclin Cdc13 [21].

To test the possibility that Spo5 may bind to $p c r 1^{+}$ mRNA to modulate its expression/function during meiosis, we performed an electrophoresis mobility shift assay (EMSA) using recombinant Spo5 protein and pcr $1^{+}$RNA transcribed in vitro. As shown in Figure 5A, the C-terminal part of Spo5, including the two RRMs (Spo5C, aas 192-567), fused to glutathione $S$-transferase (GST) (GST-Spo5C), associated with the $p c r 1^{+}$RNA (red arrowhead), but not with the control GFP RNA. To confirm direct interaction of Spo5 and $p c r 1^{+}$RNA in vivo, we performed an RNA-immunoprecipitation assay using the spo5-GFP diploid strain. Immunoprecipitation by anti-GFP antibody indicated that $p c r 1^{+}$mRNA formed a complex with Spo5-GFP in vivo, but that the mRNAs of other ATF/CREB factors did not (Figure 5B). To further confirm the specificity of binding, we compared binding of Spo5C to $p c r 1^{+}$RNA and atf $21^{+}$RNA transcribed similarly in vitro. While Spo5C bound to atf $21^{+}$RNA to some extent (Figure $5 \mathrm{C}$, lanes 7 and 8), probably because of its weak non-specific affinity for RNA, which we noticed previously [21], it was clear that $\mathrm{Spo5C}$ could bind to $p c r 1^{+}$RNA more strongly than atf $21^{+}$RNA, as $50 \mathrm{ng}$ of the protein was enough to shift $p c r 1^{+}$RNA (Figure $5 \mathrm{C}$, lane 2). We also confirmed that Spo5C carrying the F341A and F427A mutations, designated Spo5C(FAFA), lost the binding ability to $p c r 1^{+}$RNA (Figure 5D, compare lanes 2 vs. 7, and lanes 3 vs. 8), demonstrating the importance of the RRMs for the binding. These observations indicate that $p c r 1^{+}$mRNA is one of the critical targets of Spo5. We 


\section{B}
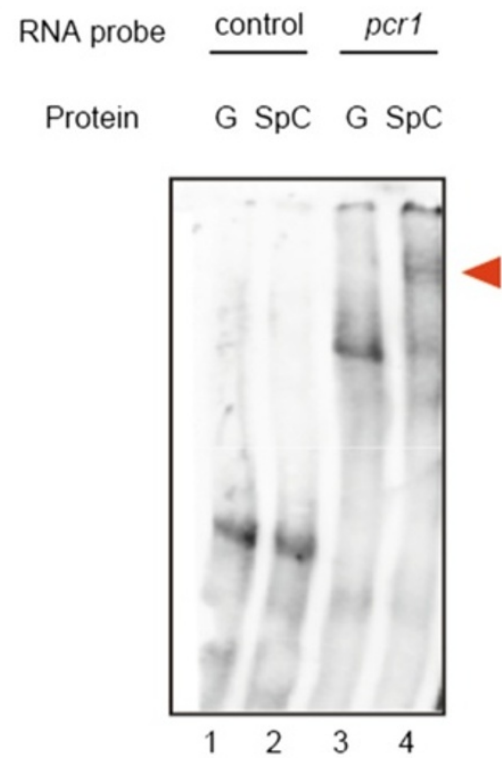

Spo5
-GFP

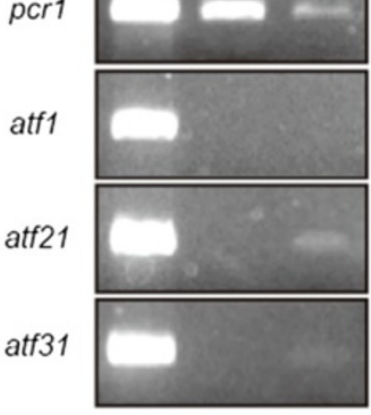

C

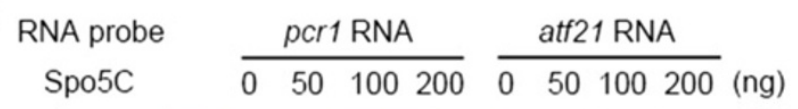

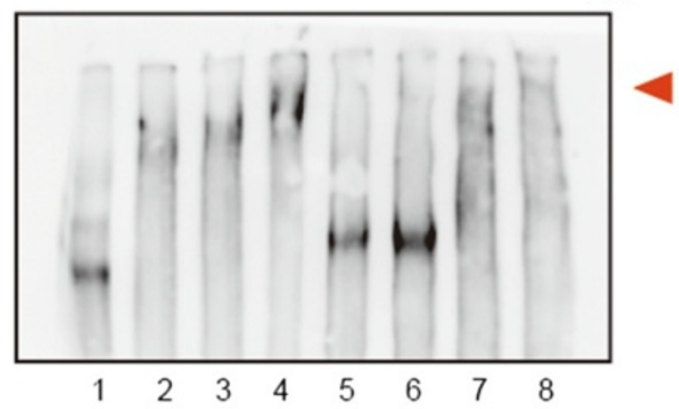

D
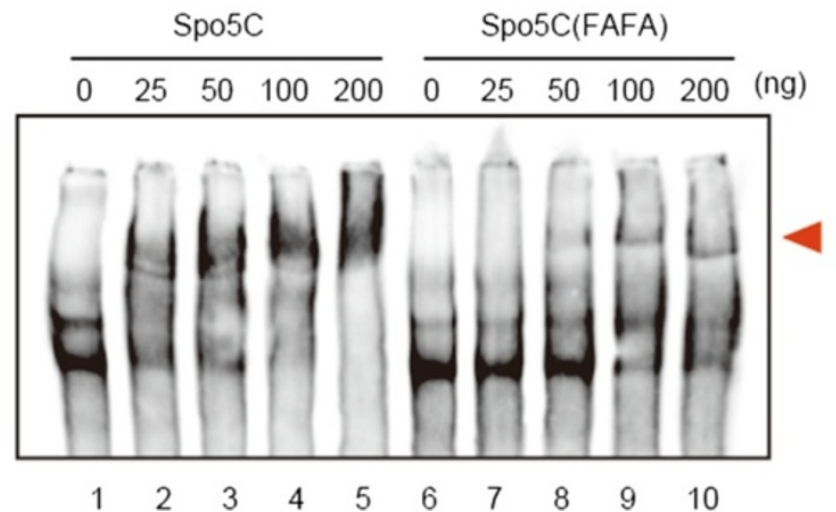

Figure $\mathbf{5}$ (See legend on next page.) 
(See figure on previous page.)

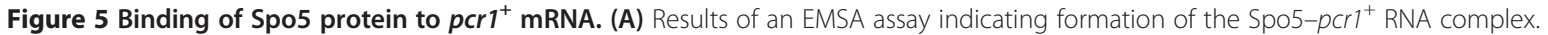
Recombinant GST and GST-Spo5C (the C-terminal part of Spo5, aas 192-567) proteins were incubated with pcr1 ${ }^{+}$RNA including the coding region and both 5'- and 3'-UTRs (lanes 3 and 4) or control GFP RNA (lanes 1 and 2). 'G' indicates GST (50 ng), and 'SpC' indicates GST-Spo5C (20 ng). The red arrowhead indicates shifted RNA. (B) Spo5-GFP and pcr $1^{+}$mRNA form complexes in vivo. A cell extract was prepared from a diploid Spo5-GFP strain undergoing meiosis and treated with anti-GFP and the control anti-HA. Reverse transcription-polymerase chain reaction (RT-PCR) assay using the pull-downs was performed to detect $p c r 1^{+}$and other ATF/CREB factors, atf1 ${ }^{+}$, atf2 $1^{+}$, and atf3 $1^{+}$mRNA. (C) $p c r 1^{+}$RNA complexes with Spo5C more efficiently than atf2 $1^{+}$RNA does. atf $21^{+}$RNA carried the coding region and both $5^{\prime}$ - and $3^{\prime}-U T R s$, similarly to $p c r 1^{+}$ RNA. The red arrowhead indicates shifted RNA. (D) Spo5C binds to pcr $1^{+}$RNA more efficiently than the mutant form Spo5C(FAFA), suggesting the involvement of the two phenylalanine residues in RNA binding. The red arrowhead indicates shifted RNA.

have shown that $c d c 13^{+}$mRNA also binds to Spo5 [21]. A previous report suggested that a long 3' UTR of $c d c 13^{+}, c d c 25^{+}$and ste $9^{+}$mRNA might determine its stability [38]. The $p c r 1^{+}$mRNA also carries a relatively long $3^{\prime}$ untranslated region (UTR), but our observation does not support the idea that Spo5 stabilizes $p c r 1^{+}$ mRNA (Additional file 4: Figure S4A). Thus, it is an interesting hypothesis to be confirmed that Spo5 may control certain activity of $p c r 1^{+}$mRNA through binding to its long $3^{\prime}$ UTR.

\section{Conclusions}

The findings of this study indicated that Spo5 is exported to the cytoplasm via the mRNA export machinery, but not via the Ran-exportin/Crm1 system. The binding of Spo5 to mRNAs through its two RRMs appears to enable its nuclear export, and this RNAbinding activity is essential not only for the nuclear export, but also for its function in the cytoplasm. Spo5 is unlikely to be involved in general mRNA export, suggesting that it may instead be involved in the posttranscriptional regulation occurring in the cytoplasm, such as control of mRNA stability and/or translational initiation. Furthermore, we identified a novel binding target of Spo5, $p c r 1^{+}$mRNA. Overexpression of the $p c r 1^{+}$ gene suppressed the sporulation deficiency of spo5 mutants, demonstrating that $p c r 1^{+}$mRNA is a critical target of Spo5. However, Pcr1 suppressed the defect in spo5 $\Delta$ cells less efficiently than Spo5, suggesting that Spo5 may also regulate factors important for meiotic progression and sporulation other than Pcr1. Suppression of spo5 by overexpression of $c d c 13^{+}$is also partial [21]. Future studies are required to identify other important targets of Spo5 and the possible role that Spo5 plays in the post-transcriptional regulation of $p c r 1^{+}$ mRNA and other mRNAs.

\section{Methods}

\section{Yeast strains and genetic manipulations}

The $S$. pombe strains used in this study are listed in Table 1. Conventional methods were used to construct gene-disrupted strains, fluorescent protein-tagged strains $[39,40]$, and a multiple fluorescent protein-tagged strain [41]. To enforce the localization of Spo5 to the nucleus and cytoplasm, the nuclear localization signal (NLS; PKKKRKV) of SV40 large T antigen and the leucine-rich nuclear export signal (NES; ILPPLERLTL) of HIV-1 Rev were used, respectively [42-44]. Standard methods were employed to grow yeast strains [45]. To induce mating, meiosis, and sporulation of homothallic $\left(h^{90}\right)$ strains, sporulation agar (SPA) or synthetic sporulation medium (SSA) was used. Haploid cells grown in liquid yeast extract containing 3\% glucose (YE) supplemented with adenine (YEA) were harvested, spotted onto SPA plates, and then incubated at $30^{\circ} \mathrm{C}$ (Figures $1,2 \mathrm{D}$ and 3 , and Additional file 2: Figure S2) and $25^{\circ} \mathrm{C}$ (Additional file 3: Figure S3B, C). Figure 4A-C illustrates the cells that were streaked on SSA plates and incubated at $30^{\circ} \mathrm{C}$. For experiments with temperature-sensitive mutants, cells were spotted onto $\mathrm{SPA}$ and incubated at $25^{\circ} \mathrm{C}$, and then transferred to $36^{\circ} \mathrm{C}$ (Figure 2A,E, Additional file 3: Figure S3A). The sporulation efficiency was calculated by counting more than 500 cells under the microscope, and each experiment was repeated three times. We also used $h^{+} / h^{-}$diploid cells (Figures 2B,C and 5B and Additional file 4: Figure S4).

\section{Mutagenesis}

To isolate novel spo5 missense mutants, the parental spo5-GFP-kan strain, in which the GFP-Tadh-kan fragment was inserted at the chromosomal $s p o 5^{+}$locus, was constructed. Genomic DNA was isolated from this strain, and a DNA fragment containing the entire coding region of $s p o 5^{+}$with GFP-Tadh1-kan genes flanked by 500-bp up- and down-stream sequences, was amplified. The amplified fragment was then subjected to errorprone PCR amplification in order to introduce random mutations to the product, using Ex Taq DNA polymerase (Takara Bio; Japan) with 40 rounds of thermal cycling. The mutagenized fragment was introduced to the homothallic $\left(h^{90}\right)$ wild-type strain JY878, and colonies that conferred G418 resistance were selected. Colonies were then replica-plated to SSA plates, and those that were deficient in sporulation were chosen using iodine staining. To exclude nonsense mutants, GFP fluorescence was monitored and GFP-positive colonies were selected. Standard sequencing methods (using a 3130 Genetic Analyzer; Applied Biosystems) were used to determine the mutation sites (F341L and S365P). The 
Table 1 Strains used in this study

\begin{tabular}{ll}
\hline No. & Genotype \\
\hline NT4 & $h^{90}$ spo5(S365P)-GFP-kan ade6-M216 leu1 ura4 \\
NT92 & $h^{90}$ spo5(F341L)-GFP-kan ade6-M216 leu1 ura4 \\
NT180 & $h^{90}$ spo5-GFP-kan mei2-mCherry-hph ade6-M216 leu1 \\
NT184 & $h^{90}$ spo5-GFP-kan ade6-M216 leu1 ura4 \\
NT196 & $h^{90}$ spo5(S365P)-GFP-kan cut11-4mRFP- $h$ h CFP-atb2-nat ade6-M216 leu1 ura4 \\
NT205 & $h^{90}$ spo5(1-296)-GFP-kan ade6-M216 leu1 ura4 \\
NT207 & $h^{90}$ spo5(1-366)-GFP-kan ade6-M216 leu1 ura4 \\
NT209 & $h^{90}$ spo5(1-456)-GFP-kan ade6-M216 leu1 ura4 \\
NT403 & $h^{90}$ spo5-GFP-kan cut11-4mRFP-hph CFP-atb2-nat ade6-M216 leu1 ura4
\end{tabular}

NT648 $\quad h^{90}$ spo5(1-525)-GFP-kan ade6-M216 leur ura4

NT716 $\quad h^{90}$ spo5(1-525)-GFP-kan cut11-4mRFP-hph CFP-atb2-nat ade6-M216 leu1 ura4

NT745 $\quad h^{90}$ spo5(F341L)-GFP-kan cut11-4mRFP-hph CFP-atb2-nat ade6-M216 leu1 ura4

NT798 $\quad h^{90}$ spo5(1-296)-GFP-kan cut11-4mRFP-hph CFP-atb2-nat ade6-M216 leu1 ura4

NT799 $\quad h^{90}$ spo5(1-366)-GFP-kan cut11-4mRFP-hph CFP-atb2-nat ade6-M216 leu1 ura4

NT814 $\quad h^{90}$ spo5(1-456)-GFP-kan cut11-4mRFP-hph CFP-atb2-nat ade6-M216 leu1 ura4

NT973 $\quad h^{90}$ spo5(RRM14 N297-366)-GFP-kan ade6-M216 leu1 ura4

NT974 $\quad h^{90}$ spo5(RRM24 $\left.\Delta 385-456\right)$-GFP-kan ade6-M216 leu1 ura4

NT1001 $\quad h^{90}$ spo5(RRM14 N297-366)-GFP-kan cut11-4mRFP-hph CFP-atb2-nat ade6-M216 leu1 ura4

NT1002 $\quad h^{90}$ spo5(RRM2 $\triangle$ 385-456)-GFP-kan cut11-4mRFP-hph CFP-atb2-nat ade6-M216 leu1 ura4

NT1168 $h^{+} / h^{-}$spo5-GFP-kan/ spo5-GFP-kan ade6-M216/ade6-M210 leu1/leu1 ura4/ura4

NT1208 $\quad h^{90}$ spo5(S365P)-GFP-kan rae1-167 ade6-M216 leu1 ura4

NT1495 $\quad h^{90}$ spo5::ura4 ${ }^{+}: .5 p 05(F 427 A)-G F P-k a n$ ade6-M216 leu1 ura4

NT1568 $\quad h^{90}$ spo5::ura4 ${ }^{+}$ade6-M216 leu1 ura4

NT1622 $h^{90}$ spo5::Ura4 ${ }^{+}: . s p 05(F 427 A)-G F P-k a n$ cut11-4mRFP-hph CFP-atb2-nat ade6-M216 leu1 ura4

NT1632 $\quad h^{90}$ spo5::ura4 ${ }^{+: . s p o 5(F 341 A)-G F P-k a n ~ a d e 6-M 216 ~ l e u 1 ~ u r a 4 ~}$

NT1634 $h^{90}$ spo5::ura4 ${ }^{+}: .5 p 05(F 341 A$, F427A)-GFP-kan ade6-M216 leu1 ura4

NT1641 $\quad h^{90}$ spo5::ura4 ${ }^{+} .:$spo5(F341A)-GFP-kan cut11-4mRFP-hph CFP-atb2-nat ade6-M216 leu1 ura4

NT1648 $\quad h^{90}$ spo5::ura4 ${ }^{+}:$. spo5(F341A, F427A)-GFP-kan cut11-4mRFP-hph CFP-atb2-nat ade6-M216 leu1 ura4

NT1677 $\quad h^{90}$ spo5-GFP-kan rae1-167 ade6-M216 leu1 ura4

NT1743 $\quad h^{90}$ spo5 + NLS-GFP-kan ade6-M216 leu1 ura4

NT1769 $h^{90}$ spo5-GFP-kan cut11-4mRFP-hph rae1-167 ade6-M216 leu1

NT1793 $h^{90}$ pabp-GFP-kan spo5:: ura4 ${ }^{+}$cut11-4mRFP-hph ade6-M216 leu1 ura4

NT1898 $h^{90}$ spo5(S365P)-GFP-kan rae1-167 cut11-4mRFP-hph ade6-M216 leu1 ura4

NT1905 hol habp-GFP-kan rae1-167 cut11-4mRFP-hph ade6-M216 leu1 ura4

NT1922 $h^{90}$ spo5(RRM14) + NES-GFP ade6-M216 leu1 ura4

NT1943 $h^{90}$ spo5(RRM1A) + NES-GFP cut11-4mRFP-hph ade6-M216 leu1 ura4

NT2130 $h^{+} / h^{-}$ade6-M216/ade6-M210 leu1/leu1 ura4/ura4

NT2132 $h^{+} / h^{-}$spo5::ura4 $4^{+} /$spo5::ura4 ${ }^{+}$ade6-M216/ade6-M210 leu1/leu1 ura4/ura4

\section{Figures}

Figure 1D, Figure $4 A B$ and Additional file 3: Figure S3BC

Figure 1D, Figure 4B and Additional file 3: Figure S3BC

Figure 2D

Figure 1BD, Figure 3A-CE and Additional file 3: Figure S3BC

Figure $1 \mathrm{C}$

Figure $1 \mathrm{~B}$ and Additional file 1: Figure S1

Figure $1 \mathrm{~B}$

Figure 1B

Figure 1AC, Figure 2A and Figure 3D,

Additional file 1: Figure S1, Additional file 2: Figure S2 and Additional file 3: Figure S3A

Figure 1B

Figure $1 \mathrm{~A}$

Figure $1 \mathrm{C}$

Figure $1 \mathrm{~A}$

Figure 1A

Figure 1A

Figure $1 \mathrm{~B}$ and Figure 3E

Figure $1 \mathrm{~B}$ and Additional file 1: Figure S1

Figure $1 \mathrm{~A}$ and Figure 3D

Figure 1A

Figure 2BC and Figure 5B

Additional file 1: Figure S1

Figure 1D

Figure $4 \mathrm{BC}$ and Additional file 3: Figure S3BC

Figure $1 \mathrm{C}$

Figure 1D

Figure 1D and Additional file 1: Figure S1

Figure $1 \mathrm{C}$

Figure $1 \mathrm{C}$ and Additional file 2: Figure S2

Additional file 1: Figure S1

Figure $3 \mathrm{~A}-\mathrm{C}$ and Additional file 1: Figure S1

Figure 2A and Additional file 3: Figure S3A

Figure 2E

Additional file 3: Figure S3A

Figure 2E

Figure $3 \mathrm{E}$

Figure 3D

Additional file 4: Figure S4A-C Additional file 4: Figure S4A-C

The original rae1-167 strain was provided by Ravi Dhar [25]. 
PrimeSTAR mutagenesis kit (Takara Bio) was used to introduce the F341A, F427A, and F341A mutations into the $\operatorname{spo~}^{+}$gene cloned using the vector pCR2.1-TOPO (Life Technologies; CA, USA). The mutated fragments were then introduced into the spo5::ura4 ${ }^{+}$strain, in which the spo5 coding region was replaced with the $u \mathrm{ra}^{+}$cassette. Colonies with correct inserts were selected on YEA plates containing $1 \mathrm{mg} / \mathrm{mL}$ 5-fluoro-orotic acid (5-FOA; Wako Pure Chemicals; Japan).

\section{Plasmids}

The plasmids containing the $p c r 1^{+}$gene $\left(\mathrm{pREP} 3-p c r 1^{+}\right)$ and the $s p o 5^{+}$gene $\left(\mathrm{pREP} 3-\mathrm{spo5^{+ }}\right.$ ) were isolated from a cDNA library. The pREP1 plasmids carrying the atf $1^{+}$, atf $21^{+}$, and atf $31^{+}$genes were a gift from Takatomi Yamada [37], and we subsequently replaced their $n m t 1$ promoters with the $n m t 81$ promoter.

\section{Microscopy}

An Axioplan2 fluorescence microscope (Zeiss; Germany) equipped with a CoolSNAP HQ2 CCD camera (Photometrics; AZ, USA) and SlideBook software (Leeds Precision; $\mathrm{MN}, \mathrm{USA}$ ) were used to acquire the images presented in Figures 2, 3A,B and 4A, Additional file 2: Figure S2 and Additional file 3: Figure S3A. Single-sectioned images along the Z-axis were captured and deconvolved. Single-cell imaging was performed using the DeltaVision-SoftWoRx system (GE Healthcare; UK) with a CoolSNAP HQ2 CCD camera, as described previously [41]. Briefly, the cells selected for observation were mounted on a glass-bottomed dish (Matsunami Glass; Japan) precoated with lectin and filled with $\mathrm{MM}-\mathrm{N}$ liquid medium. Serial-sectioned images were acquired along the $\mathrm{Z}$-axis and stacked using the 'quick projection' algorithm in SoftWoRx.

To block the Crm1/exportin-dependent nuclear export machinery, $100 \mathrm{ng} / \mathrm{mL}$ leptomycin B (LMB) [28] was added to a culture of Spo5-GFP Mei2-mCherry cells. After $60 \mathrm{~min}$ incubation at $30^{\circ} \mathrm{C}$, cells were subjected to fluorescence microscopy. Mei2-mCherry, an LMB-sensitive meiotic protein, served as a positive control [46].

The rae1-167 strain was a gift from Ravi Dhar [25]. We employed this mutant to arrest mRNA export during meiosis. Cells were incubated on SPA at $25^{\circ} \mathrm{C}$ for $6 \mathrm{~h}$, and then shifted to a restrictive temperature at $36^{\circ} \mathrm{C}$ for $3 \mathrm{~h}$ prior to microscopic observation.

\section{Electrophoretic mobility shift assay (EMSA) of RNA}

As reported previously [47], digoxigenin (DIG)-labeled RNA was prepared from PCR products using the DIG RNA Labeling kit (SP6/T7) (Roche). The RNA-binding reaction was performed using 2 ng of DIG-labeled RNA (control; derived from the GFP sequence, pcr1 and atf21) and 20-200 ng of either recombinant GST, GST-Spo5C (aas 192-567), or GST-Spo5C(FAFA), in $4 \mu \mathrm{L}$ of a modified
KNET buffer consisting of $20 \mathrm{mM} \mathrm{KCl}, 80 \mathrm{mM} \mathrm{NaCl}$, $2 \mathrm{mM}$ ethylene glycol bis-(2-aminoethylether) tetraacetic acid (EGTA), $50 \mathrm{mM}$ Tris- $\mathrm{HCl}$ (pH7.5), 0.05\% NP-40, $1 \mathrm{mM} \mathrm{MgCl}_{2}$, $2 \mathrm{mM}$ dithiothreitol, 10\% glycerol, and RNase Inhibitor (Roche). RNA with the GFPcoding sequence was used as a negative control. Samples were preincubated at room temperature with $10 \mu \mathrm{g}$ of carrier Escherichia coli tRNA for 25 min. Labeled RNA was then added and incubated for another $25 \mathrm{~min}$. Samples were analyzed using polyacrylamide gel electrophoresis and electroblotted to a GeneScreen Plus membrane (NEN) using $0.5 \times$ Tris-borate-EDTA (TBE) buffer. Signals were detected using a DIG Luminescent Detection Kit (Roche).

\section{RNA-immunoprecipitation}

Diploid cells expressing Spo5-GFP were cultured in $\mathrm{MM}+\mathrm{N}$ at $30^{\circ} \mathrm{C}$ for $15 \mathrm{~h}$, shifted to MM-N $\left(0 \mathrm{~h}, 30^{\circ} \mathrm{C}\right)$ and sampled for RNA extraction after $6 \mathrm{~h}$. Detailed conditions for RNA-IP were described previously [48]. Immunoprecipitated RNA was isolated by phenol-chloroform extraction. For immunoprecipitation, anti-GFP (Roche; GFP monoclonal antibody) and anti-HA (Abcam; 16B12, HA monoclonal antibody) were used. Reverse transcription was performed using the TaKaRa RNA PCR kit (AMV, Ver 3.0; Takara bio).

\section{Other methods}

Western blotting, reverse transcription and quantitative PCR were performed according to the same protocols as we described previously [21]. Following oligonucleotides were used in RT-qPCR: pcr1(forward) CCGAAT TCTGGAGCGCAAT, pcr1(reverse) CACTCTTTCTTTT TCTGGCGAAA, act1(forward) TGAGGAGCACCCT TGCTTGT, and act1(reverse) TCTTCTCACGGTT GGATTTGG. Inhibition of mRNA transcription by 1,10-phenanthroline was carried out as described precisely by Galipon et al. [49].

\section{Availability of supporting data}

The data sets supporting the results of this article are included within the article and its additional files.

\section{Additional files}

Additional file 1: Figure S1. Expression level of mutant Spo5 proteins under meiotic conditions. Cell extracts were prepared from representative spo 5 mutant strains incubated on SPA at $25^{\circ} \mathrm{C}$ for 8 hours. They were separated by SDS-PAGE and subjected to western blotting. Each Spo5-GFP protein, which was expressed from the authentic spo5 promoter, was detected with an anti-GFP antibody. a-tubulin was also detected as a loading control. The wild-type strain shown here for comparison carried an additional gene encoding CFP-tagged Atb2, which reacted with both anti-GFP and anti-a-tubulin (lane 1). 
Additional file 2: Figure S2. Localization of Spo5(FAFA) protein during the progression of meiosis. Localization of WT Spo5 (left) and Spo5(FAFA) (right) was examined at four stages of meiosis, namely horsetail-movement, one-nucleus, two-nuclei, and four-nuclei stages. GFP-tagged Spo5 (green) and the nuclear envelope marker Cut11-4mRFP (red) were detected. Scale bar, $5 \mu \mathrm{m}$.

Additional file 3: Figure S3. Characterization of a novel spo5 missense mutant spo5(S365P). (A) Spo5(S365P)-GFP did not accumulate in the nucleus of the temperature-sensitive rae1-167 cells. Cells were incubated at $25^{\circ} \mathrm{C}$ for $6 \mathrm{~h}$ and then shifted to $36^{\circ} \mathrm{C}$ for $3 \mathrm{~h}$. Cut $11-4 \mathrm{mRFP}$ was used as a nuclear envelope marker. (B) At $25^{\circ} \mathrm{C}, W T$ and spo5(F341L) cells were stained dark brown with iodine vapor and sporulated efficiently, while spo5 (S365P) cells were stained light brown and sporulated weakly. The spo5 $\Delta$ cells showed a white colony and did not sporulate. At $30^{\circ} \mathrm{C}$, the two mutants showed nearly white colonies and did not sporulate efficiently, as indicated quantitatively in Figure 1D. (C) Sporulation efficiency of WT and the two spo5 mutant strains at $25^{\circ} \mathrm{C}(\mathrm{n}>500)$. Error bars indicate standard deviation.

Additional file 4: Figure S4. Expression of $\mathrm{crr}^{+} \mathrm{mRNA}$ is not lowered in spo5 $\Delta$ cells. (A) Meiosis was induced in $\operatorname{spo5}^{+}(\mathrm{WT})$ and spo5 $\Delta$ diploid cells, as in Figure 5B, and total RNA was isolated from them every 2 hours. The relative amount of $p c r 1^{+}$mRNA in $s p 05^{+}$and $s p o 5 \Delta$ cells was determined by RT-qPCR. The quantity of mRNA was normalized to the expression level of act1 mRNA. Error bars show standard deviation. Two independent samples were analyzed for each strain. $n=3$, for each sample. (B) Sporulation efficiency was measured for spo5 $\Delta$ cells harboring either the vector, the $\mathrm{pCr}^{+}$ clone, or the $s p o 5^{+}$clone. $(n>500)$ (C) The relative amount of $p c r 1^{+}$mRNA was determined by RT-qPCR in spo5 $\triangle$ cells harboring either the vector, the $\mathrm{pCr}^{+}$clone, or the $\mathrm{spo}^{+}{ }^{+}$clone. Total RNA was isolated every 4 hours after the induction of meiosis, and analyzed as in (A). Error bars show standard deviation.

\section{Competing interests}

The authors declare that they have no competing interests.

\section{Authors' contributions}

NT performed the experiments and analyzed the results. AY and MS designed the outline of this study and analyzed the results. MY supervised the work. NT, AY, MS, and MY wrote the manuscript. All authors read and approved the final manuscript.

\section{Acknowledgements}

We are grateful to Drs. Ravi Dhar and Takatomi Yamada for providing the materials. We highly appreciate assistance of Dr. Mayumi Arata and Mr. Yuichi Shichino in quantitative experiments. This work was supported by Grants-in-Aid for Specially Promoted Research and Scientific Research (S) (to M.Y.) and for Scientific Research (C) (to A.Y.) from Japan Society for the Promotion of Science (JSPS), and Grant-in-Aid for Scientific Research on Priority Areas "Cell Proliferation Control" from the Ministry of Education, Culture, Sports, Science and Technology (MEXT) (to M.S.). This work was also supported in part by the Global COE Program (Integrative Life Science Based on the Study of Biosignaling Mechanisms), MEXT, Japan.

\section{Author details}

'Kazusa DNA Research Institute, 2-6-7 Kazusa-kamatari, Kisarazu, Chiba 292-0818, Japan. ${ }^{2}$ Department of Biophysics and Biochemistry, Graduate School of Science, University of Tokyo, 7-3-1 Hongo, Tokyo 113-0033, Japan. ${ }^{3}$ Department of Life Science and Medical Bioscience, Graduate School of Advanced Science and Engineering, Waseda University, 2-2 Wakamatsucho, Shinjuku, Tokyo 162-8480, Japan. ${ }^{4}$ National Institute for Basic Biology, Nishigonaka 38, Myodaiji, Okazaki, Aichi 444-8585, Japan.

\section{Received: 18 November 2013 Accepted: 3 July 2014}

Published: 15 July 2014

\section{References}

1. van Werven FJ, Amon A: Regulation of entry into gametogenesis. Philos Trans R Soc Lond B Biol Sci 2011, 366(1584):3521-3531.

2. Handel MA, Schimenti JC: Genetics of mammalian meiosis: regulation, dynamics and impact on fertility. Nat Rev Genet 2010, 11(2):124-136.
3. Yamamoto $\mathrm{M}$, Imai $\mathrm{Y}$, Watanabe $\mathrm{Y}$ : Mating and sporulation in Schizosaccharomyces pombe. The Molecular and Cellular Biology of the yeast Sacchromyces, Cold Spring Harbor Laboratory Press 1997, 3:1037-1106.

4. Yamamoto M: The selective elimination of messenger RNA underlies the mitosis-meiosis switch in fission yeast. Proc J.Jn Acad Ser B Phys Biol Sci 2010, 86(8):788-797.

5. Mata J, Lyne R, Burns G, Bahler J: The transcriptional program of meiosis and sporulation in fission yeast. Nat Genet 2002, 32(1):143-147.

6. Mata J, Wilbrey A, Bahler J: Transcriptional regulatory network for sexual differentiation in fission yeast. Genome Biol 2007, 8(10):R217.

7. Amorim MJ, Cotobal C, Duncan C, Mata J: Global coordination of transcriptional control and mRNA decay during cellular differentiation. Mol Syst Biol 2010, 6:380.

8. Horie S, Watanabe Y, Tanaka K, Nishiwaki S, Fujioka H, Abe H, Yamamoto M, Shimoda C: The Schizosaccharomyces pombe mei4+ gene encodes a meiosis-specific transcription factor containing a forkhead DNA-binding domain. Mol Cell Biol 1998, 18(4):2118-2129.

9. Chu S, Herskowitz I: Gametogenesis in yeast is regulated by a transcriptional cascade dependent on Ndt80. Mol Cell 1998, 1(5):685-696.

10. Winter E: The Sum $1 / \mathrm{Ndt} 80$ transcriptional switch and commitment to meiosis in Saccharomyces cerevisiae. Microbiol Mol Biol Rev 2012, 76(1):1-15.

11. Chu S, DeRisi J, Eisen M, Mulholland J, Botstein D, Brown PO, Herskowitz I: The transcriptional program of sporulation in budding yeast. Science 1998, 282(5389):699-705

12. Primig M, Williams RM, Winzeler EA, Tevzadze GG, Conway AR, Hwang SY, Davis RW, Esposito RE: The core meiotic transcriptome in budding yeasts. Nat Genet 2000, 26(4):415-423.

13. Mendez R, Richter JD: Translational control by CPEB: a means to the end. Nat Rev Mol Cell Biol 2001, 2(7):521-529.

14. Harigaya Y, Tanaka H, Yamanaka S, Tanaka K, Watanabe Y, Tsutsumi C, Chikashige Y, Hiraoka Y, Yamashita A, Yamamoto M: Selective elimination of messenger RNA prevents an incidence of untimely meiosis. Nature 2006, 442(7098):45-50.

15. Bresch C, Muller G, Egel R: Genes involved in meiosis and sporulation of a yeast. Mol Gen Genet 1968, 102(4):301-306.

16. Kishida M, Shimoda C: Genetic mapping of eleven spo genes essential for ascospore formation in the fission yeast Schizosaccharomyces pombe. Curr Genet 1986, 10(6):443-447.

17. Hirata A, Shimoda C: Electron microscopic examination of sporulation-deficient mutants of the fission yeast Schizosaccharomyces pombe. Arch Microbiol 1992, 158(4):249-255.

18. Hirata A, Shimoda C: Structural modification of spindle pole bodies during meiosis II is essential for the normal formation of ascospores in Schizosaccharomyces pombe: ultrastructural analysis of spo mutants. Yeast 1994, 10(2):173-183.

19. Kasama T, Shigehisa A, Hirata A, Saito TT, Tougan T, Okuzaki D, Nojima H: Spo5/Mug12, a putative meiosis-specific RNA-binding protein, is essential for meiotic progression and forms Mei2 dot-like nuclear foci. Eukaryot Cell 2006, 5(8):1301-1313.

20. Okuzaki D, Kasama T, Hirata A, Ohtaka A, Kakegawa R, Nojima H: Spo5 phosphorylation is essential for its own timely degradation and for successful meiosis in Schizosaccharomyces pombe. Cell Cycle 2010, 9(18):3751-3760.

21. Arata M, Sato M, Yamashita A, Yamamoto M: The RNA-binding protein Spo5 promotes meiosis II by regulating cyclin $\mathrm{Cdc} 13$ in fission yeast. Genes Cells 2014, 19(3):225-238.

22. Maris $C$, Dominguez $C$, Allain FH: The RNA recognition motif, a plastic RNA-binding platform to regulate post-transcriptional gene expression. FEBS J 2005, 272(9):2118-2131.

23. Murphy R, Watkins JL, Wente SR: GLE2, a saccharomyces cerevisiae homologue of the schizosaccharomyces pombe export factor RAE1, is required for nuclear pore complex structure and function. Mol Biol Cell 1996, 7(12):1921-1937.

24. Kraemer D, Blobel G: mRNA binding protein mrnp 41 localizes to both nucleus and cytoplasm. Proc Natl Acad Sci U S A 1997, 94(17):9119-9124.

25. Brown JA, Bharathi A, Ghosh A, Whalen W, Fitzgerald E, Dhar R: A mutation in the schizosaccharomyces pombe rae1 gene causes defects in poly (A) + RNA export and in the cytoskeleton. J Biol Chem 1995, 270(13):7411-7419.

26. Watanabe M, Fukuda M, Yoshida M, Yanagida M, Nishida E: Involvement of CRM1, a nuclear export receptor, in mRNA export in mammalian cells and fission yeast. Genes Cells 1999, 4(5):291-297. 
27. Moore MS, Blobel G: The GTP-binding protein Ran/TC4 is required for protein import into the nucleus. Nature 1993, 365(6447):661-663.

28. Kudo N, Wolff B, Sekimoto T, Schreiner EP, Yoneda Y, Yanagida M, Horinouchi S, Yoshida M: Leptomycin B inhibition of signal-mediated nuclear export by direct binding to CRM1. Exp Cell Res 1998, 242(2):540-547.

29. Thakurta AG, Ho Yoon J, Dhar R: Schizosaccharomyces pombe spPABP, a homologue of Saccharomyces cerevisiae Pab1p, is a non-essential, shuttling protein that facilitates mRNA export. Yeast 2002, 19(9):803-810

30. Volpi S, Bongiorni S, Fabbretti F, Wakimoto BT, Prantera G: Drosophila rae1 is required for male meiosis and spermatogenesis. J Cell Sci 2013, 126(Pt 16):3541-3551.

31. Burgess HM, Richardson WA, Anderson RC, Salaun C, Graham SV, Gray NK: Nuclear relocalisation of cytoplasmic poly (A)-binding proteins PABP1 and PABP4 in response to UV irradiation reveals $m R N A$-dependent export of metazoan PABPs. J Cell Sci 2011, 124(Pt 19):3344-3355.

32. Zhang T, Delestienne N, Huez G, Kruys V, Gueydan C: Identification of the sequence determinants mediating the nucleo-cytoplasmic shuttling of TIAR and TIA-1 RNA-binding proteins. J Cell Sci 2005, 118(Pt 23):5453-5463.

33. Cassola A, Frasch AC: An RNA recognition motif mediates the nucleocytoplasmic transport of a trypanosome RNA-binding protein. J Biol Chem 2009, 284(50):35015-35028.

34. Watanabe $Y$, Yamamoto M: Schizosaccharomyces pombe pcr1+ encodes a CREB/ATF protein involved in regulation of gene expression for sexual development. Mol Cell Biol 1996, 16(2):704-711.

35. Kanoh J, Watanabe $Y$, Ohsugi M, lino Y, Yamamoto M: Schizosaccharomyces pombe gad7+ encodes a phosphoprotein with a bZIP domain, which is required for proper G1 arrest and gene expression under nitrogen starvation. Genes Cells 1996, 1(4):391-408.

36. Wahls WP, Smith GR: A heteromeric protein that binds to a meiotic homologous recombination hot spot: correlation of binding and hot spot activity. Genes Dev 1994, 8(14):1693-1702.

37. Morita T, Yamada T, Yamada S, Matsumoto K, Ohta K: Fission yeast ATF/ CREB family protein Atf21 plays important roles in production of normal spores. Genes Cells 2011, 16(2):217-230.

38. Daga RR, Bolanos P, Moreno S: Regulated mRNA stability of the Cdk inhibitor Rum 1 links nutrient status to cell cycle progression. Curr Biol 2003, 13(23):2015-2024.

39. Bahler J, Wu JQ, Longtine MS, Shah NG, McKenzie A 3rd, Steever AB, Wach A, Philippsen P, Pringle JR: Heterologous modules for efficient and versatile PCR-based gene targeting in Schizosaccharomyces pombe. Yeast 1998, 14(10):943-951.

40. Sato M, Dhut S, Toda T: New drug-resistant cassettes for gene disruption and epitope tagging in Schizosaccharomyces pombe. Yeast 2005, 22(7):583-591

41. Sato M, Toya M, Toda T: Visualization of fluorescence-tagged proteins in fission yeast: the analysis of mitotic spindle dynamics using GFP-tubulin under the native promoter. Methods Mol Biol 2009, 545:185-203.

42. Goldfarb DS, Gariepy J, Schoolnik G, Kornberg RD: Synthetic peptides as nuclear localization signals. Nature 1986, 322(6080):641-644.

43. Wychowski C, Benichou D, Girard M: A domain of SV40 capsid polypeptide VP1 that specifies migration into the cell nucleus. EMBO J 1986, 5(10):2569-2576.

44. Wolff B, Sanglier JJ, Wang Y: Leptomycin B is an inhibitor of nuclear export: inhibition of nucleo-cytoplasmic translocation of the human immunodeficiency virus type 1 (HIV-1) Rev protein and Rev-dependent mRNA. Chem Biol 1997, 4(2):139-147.

45. Moreno S, Klar A, Nurse P: Molecular genetic analysis of fission yeast Schizosaccharomyces pombe. Methods Enzymol 1991, 194:795-823.

46. Sato M, Shinozaki-Yabana S, Yamashita A, Watanabe Y, Yamamoto M: The fission yeast meiotic regulator Mei2p undergoes nucleocytoplasmic shuttling. FEBS Lett 2001, 499(3):251-255.

47. Yamashita A, Shichino $Y$, Tanaka H, Hiriart E, Touat-Todeschini L, Vavasseur A, Ding DQ, Hiraoka Y, Verdel A, Yamamoto M: Hexanucleotide motifs mediate recruitment of the RNA elimination machinery to silent meiotic genes. Open Biol 2012, 2(3):120014.
48. Hiriart E, Vavasseur A, Touat-Todeschini L, Yamashita A, Gilquin B, Lambert E, Perot J, Shichino Y, Nazaret N, Boyault C, Lachuer J, Perazza D, Yamamoto M, Verdel A: Mmi1 RNA surveillance machinery directs RNAi complex RITS to specific meiotic genes in fission yeast. EMBO J 2012 31(10):2296-2308

49. Galipon J, Miki A, Oda A, Inada T, Ohta K: Stress-induced IncRNAs evade nuclear degradation and enter the translational machinery. Genes Cells 2013, 18(5):353-368.

doi:10.1186/1471-2180-14-188

Cite this article as: Togashi et al:: Functional significance of nuclear export and mRNA binding of meiotic regulator Spo5 in fission yeast. BMC Microbiology 2014 14:188.

\section{Submit your next manuscript to BioMed Central and take full advantage of:}

- Convenient online submission

- Thorough peer review

- No space constraints or color figure charges

- Immediate publication on acceptance

- Inclusion in PubMed, CAS, Scopus and Google Scholar

- Research which is freely available for redistribution

Submit your manuscript at www.biomedcentral.com/submit
C) BioMed Central 